\title{
Effect of Acetylsalicylic Acid on Fatty Acid $\omega$-Hydroxylation in Rat Liver
}

\author{
RICHARD OKITA \\ Department of Biochemistry, Medical College of Wisconsin, Milwaukee, Wisconsin 53226
}

\begin{abstract}
The effect of acetylsalicylic acid on the cytochrome P-450-mediated fatty acid $\omega$-hydroxylation system was assessed in male Sprague-Dawley rats after they were fed a diet containing $1 \%(w / w)$ acetylsalicylic acid. A 3-fold increase in the specific activity of laurate $\omega$-hydroxylation was observed in the acetylsalicylic acid fed rats in comparison to control rats. This effect of acetylsalicylic acid was unique as the specific activities of other cytochrome P-450-mediated reactions were not increased. The induction of the laurate $\omega$-hydroxylation system was also manifested in a rapid formation of its dicarboxylic acid derivative, dodecanedioic acid, as the $\omega$-hydroxy derivative was further oxidized by alcohol and aldehyde dehydrogenases. These results suggest the acetylsalicylic acid is similar to other peroxisomal proliferating agents in that it also induces the microsomal fatty acid $\omega$-hydroxylation system and may account for the appearance of unique dicarboxylic acids in Reye's syndrome patients. (Pediatr Res 20: 12211224, 1986)
\end{abstract}

Abbreviations
ASA, acetylsalicylic acid
BP, benzphetamine
DCA, dicarboxylic acid
DEHP, di(2-ethylhexyl)phthalate
ECOD, ethoxycoumarin
EROD, ethoxyresorufin
11-OH-LA, 11-hydroxylauric
12-OH-LA, 12-hydroxylauric acid
LA, lauric acid
RS, Reye's syndrome

A recent study by Tonsgard and Getz (1) reported that over $50 \%$ of the free fatty acids in the serum of RS patients were DCAs and it was postulated that the DCAs were involved in the impairment of mitochondrial function that is associated with this disease. DCAs were observed to uncouple mitochondrial oxidative phosphorylation and to cause mitochondria to lose their condense structure and assume a swollen appearance (1). Ninety percent of the dicarboxylic acids in the RS patients were identified as containing 16- or 18-carbon atoms. $\mathrm{Ng}$ et al. (2) initially had reported these unusual long chain DCAs in RS patients in addition to adipic, suberic, and sebacic acids.

DCAs are found in minute quantities in the urine or serum of healthy individuals (3), but they become elevated in individuals

Received March 5, 1986: accepted July 2, 1986.

Correspondence and reprints Richard Okita, Ph.D., Department of Biochemistry. The Medical College of Wisconsin, 8701 Watertown Plank Road, Milwaukee, WI 53226 .

This study was supported in part by PHS Grants BRSG S07 RRO5434, AG 04856, and ES 03771 . that are ketotic (4) or in individuals that have an impaired fatty acid $\beta$-oxidation system such as in glutaric aciduria type II $(5$, 6 ), ethylmalonic-adipic aciduria (7), systemic carnitine deficiency (8), hypoglycin toxicity (9), acyl-CoA dehydrogenase deficiency $(10,11)$, and in $\operatorname{RS}(1,2)$. The biochemical basis of RS has not been delineated and multiple etiological factors may be responsible for this syndrome (11). Fatty acids have been implicated as a factor in RS (11). The formation of DCAs is caused by the oxidation of the methyl carbon atom of fatty acids to a carboxyl group and is catalyzed by a series of enzymes that are referred to as the fatty acid $\omega$-oxidation system. The initial and rate-limiting step in this sequence of reactions is catalyzed by a microsomal cytochrome P-450-mediated hydroxylase which produces an $\omega$ hydroxy-fatty acid (12-14). This derivative is further oxidized by cytosolic alcohol and aldehyde dehydrogenases to form the DCA $(15,16)$.

The cytochrome P-450 that catalyzes the $\omega$-hydroxylation reaction was considered to be a constitutive, noninducible member of the cytochrome P-450 family of isozymes (17), but recent studies have demonstrated that clofibrate $(18,19)$ and DEHP $(20,21)$, potent peroxisomal proliferating agents, are also inducers of the hepatic fatty acid $\omega$-hydroxylation system.

ASA has been reported to be another peroxisomal proliferator and inducer of its fatty acid $\beta$-oxidation system $(22,23)$, but it has not been established if it is an inducer of the microsomal fatty acid $\omega$-hydroxylation system. Several studies have indicated that the development of RS is linked to ASA therapy (24-26). Because of the possible inductive action of ASA on the $\omega$ hydroxylation system and the observation that DCAs in the serum of RS patients are mitochondrial inhibitors, ASA was administered to rats to determine its effect on the fatty acid $\omega$ hydroxylation system and in the formation of the DCAs using the 12-carbon atom fatty acid, LA, as substrate.

\section{MATERIALS AND METHODS}

Male Sprague-Dawley rats (150 g) were purchased from King Animals (Madison, WI) and were fed a standard Purina Rat Chow diet (St. Louis, MO) for 1 or 2 wk to allow them to acclimate to their surroundings. Rats were then placed on an ASA-supplemented diet $(1 \% \mathrm{w} / \mathrm{w}$ of rat food) prepared in pelleted form by Ralston Purina (Richmond, IN) for 2 wk to induce the peroxisomal palmitoyl-CoA oxidation system as described by Ishii and Suga (22). Control rats were continued on the standard rat Chow. Food was withdrawn 15- to 18 -h prior to sacrificing the rats. The microsomal fraction was prepared from livers by the procedures described by Okita et al. (27). The effects of ASA-feeding on laurate $\omega$ - or 12- and ( $\omega-1)$ - or 11-hydroxylation reactions was examined by the method of Okita and Chance (20) using trace quantities of $\left[1-{ }^{14} \mathrm{C}\right]$-LA (Amersham, Arlington Heights, IL) to follow the separation of the 11- and 12-hydroxylated (11-OH and 12-OH) derivatives by high-pressure liquid chromatography on a Bio Rad ODS-5 $(15 \times 0.4 \mathrm{~cm})$ reverse phase column (Richmond, CA). 
The formation of the DCA derivative, dodecanedioic acid, was assayed in the $17,400 \times g$ liver supernatants of control rats or rats treated with ASA for $2 \mathrm{wk}$. The reactions were performed in $55 \mathrm{mM}$ potassium phosphate buffer containing $10 \mathrm{mM} \mathrm{MgCl}$, $1 \mathrm{mM}$ NADPH, and $1 \mathrm{mM} \mathrm{NAD}^{+}, 100 \mu \mathrm{M}$ sodium laurate (Sigma, St. Louis, MO) with $0.1 \mu \mathrm{Ci}\left[1-{ }^{14} \mathrm{C}\right]$ lauric acid, $8 \mathrm{mM}$ isocitrate (Sigma, St. Louis, MO) with $0.1 \mathrm{mg}$ isocitrate dehydrogenase (Boehringer-Mannheim, Indianapolis, IN), and $5 \mathrm{mg}$ of the $17,400 \times g$ supernatant fraction. The reactions were performed at $37^{\circ} \mathrm{C}$ for $10 \mathrm{~min}$ and the reactions terminated by adding $0.2 \mathrm{ml}$ of $1 \mathrm{~N} \mathrm{HCl}$ and extracting with ethylacetate as described by Okita and Chance (20) for the laurate hydroxylation assays. The retention times for the $12-\mathrm{OH}$ and $11-\mathrm{OH}$ derivatives of laurate and the DCA were $15.5,13.8$, and $12.7 \mathrm{~min}$, respectively. The identity of the hydroxylated LA derivatives and of the dodecanedioic acid were confirmed by cochromatography with standards using a refractive index monitor and by gas chromatography-mass spectral analysis of the trimethyl silylatedderivatized samples. The $12-\mathrm{OH}$ and dicarboxylic acid derivatives of lauric acid are available from Sigma. The 11-OH-LA was produced enzymatically using liver microsomes from phenobarbital-treated rats and a NADPH generating system.

The benzphetamine N-demethylation reaction was performed by the procedure of Werringloer (28) and the ethoxycoumarin and ethoxyresorufin O-deethylation reactions were assayed by the procedure of Prough et al. (29). Peroxisomal-mediated palmitoyl-CoA oxidation was examined in liver homogenates (centrifuged at $1090 \times g$ to remove unbroken cells and cell debris) by the procedure of Lazarow (30). The oxidation of the 12-OHLA (Sigma) by NAD ${ }^{+}$-dependent alcohol and aldehyde dehydrogenases was assayed by following the increase in absorbance at $340 \mathrm{~nm}$ in the $105,000 \times g$ supernatant fraction (31). No measurable oxidation of 12-OH-LA was observed using the microsomal fraction, so only the $105,000 \times g$ supernatant was used.

\section{RESULTS}

The addition of ASA to the diet of male Sprague-Dawley rats at a concentration of $1 \%(\mathrm{w} / \mathrm{w})$ for $1 \mathrm{wk}$ caused a 3 -fold stimulation in the peroxisomal mediated, cyanide-insensitive palmitoyl-CoA oxidation system (Table 1). A 3-fold increase in the microsomal cytochrome P-450-mediated LA $\omega$-hydroxylase was also observed (Table 1). These data are consistent with previous reports that peroxisomal proliferating agents also are inducers of the microsomal fatty acid $\omega$-hydroxylation system. This ASAmediated induction of the cytochrome P-450 monooxygenase is highly specific for the species that catalyzes the $\omega$-hydroxylation of fatty acids, as the feeding of ASA did not stimulate the specific activities of substrates that are metabolized by other isozymes of cytochrome P-450. As shown in Table 2, the specific activities of benzphetamine $\mathrm{N}$-demethylation, ethoxycoumarin O-deethy-

Table 1. Effect of ASA-feeding on peroxisomal-mediated palmitoyl-CoA oxidation and microsomal-mediated laurate 12-hydroxylation

\begin{tabular}{ccc}
\hline & \multicolumn{2}{c}{ Reaction } \\
\cline { 2 - 3 } & \multicolumn{2}{c}{$\begin{array}{c}\text { Laurate } 12-\text { hydroxylation } \\
\text { (nmol 12-OH-LA/min/ } \\
\text { Microsomes }\end{array}$} \\
\hline Control & (nmol NADH/min/mg) & $\begin{array}{c}\mathrm{mg} \text { ) } \\
\text { ASA }\end{array}$ \\
\hline
\end{tabular}

* The values represent the mean of three experiments \pm SD. The livers of four rats were pooled to prepare the homogenates for each experiment. The values are expressed as $\mathrm{mg}$ of the homogenate fraction.

$\dagger$ The values represent the means of three experiments in which the livers of three or four rats were pooled to prepare the microsomal fraction. The values are expressed as $\mathrm{mg}$ microsomal protein.
Table 2. Effect of ASA-feeding on cytochrome P-450 monooxygenase activities in rat liver microsomes (Mean $\pm S D$ )

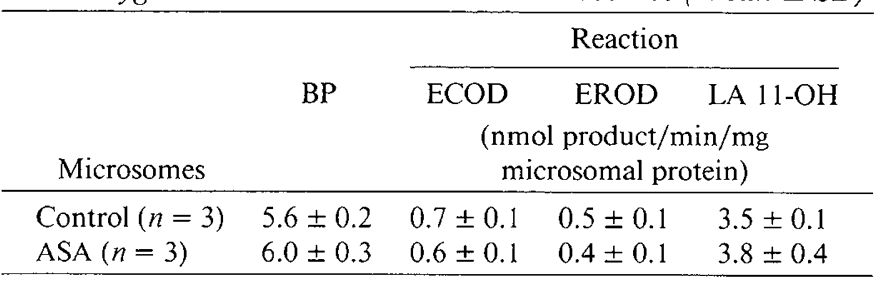

Table 3. Percent of radioactivity as LA and its w-oxidized metabolites in rat liver postmitochondrial supernatant fractions

\begin{tabular}{lrcccc}
\hline & LA & 12-OH-LA & 11-OH-LA & DCA & Other metabolites \\
\cline { 2 - 6 } \multicolumn{5}{c}{ Rat } & \multicolumn{5}{c}{$\%$ distribution } \\
\hline Control & 65 & 5 & 20 & 10 & 12 \\
ASA & 1 & ND* $^{*}$ & ND & 87 & 12 \\
\hline
\end{tabular}

* Not detected.

Table 4. Effect of $A S A$ on the rat liver cytosolic $N A D^{+}$dependent 12-hydroxy-lauric acid oxidation*

\begin{tabular}{cc}
\hline Rat & $\begin{array}{c}\text { 12-OH-LA oxidation } \\
\text { (nmol NADH reduced } / \mathrm{min} / \mathrm{mg})\end{array}$ \\
\hline Control $(n=3)$ & $11.9 \pm 0.8$ \\
ASA $(n=3)$ & $14.7 \pm 0.9$ \\
\hline
\end{tabular}

* The values represent the mean $\pm \mathrm{SD}$. Values are expressed as mg of the $105,000 \times g$ supernatant fraction.

lation, ethoxyresorufin O-deethylation, and laurate 11-hydroxylation were not increased by the ASA feeding.

The induction of the cytochrome P-450-mediated $\omega$-hydroxylase by ASA caused the rapid utilization of LA and led to the formation of its DCA derivative in assays performed with postmitochondrial supernatant fractions (Table 3). The postmitochondrial supernatant fraction $(17,400 \times g)$ from control-fed rats metabolized $35 \%$ of the lauric acid in 10 min of which $10 \%$ was found as dodecanedioic acid, $20 \%$ as $11-\mathrm{OH}-\mathrm{LA}$ and $5 \%$ as $12-$ OH-LA. In the aspirin-fed rats, only $1 \%$ of the radioactivity remained as the unmetabolized lauric acid and $87 \%$ was metabolized to the DCA derivative in this time period. These studies demonstrated that aspirin feeding stimulated the formation of the 12-OH-LA and the rapid oxidation to its dicarboxylic acid. The effect of aspirin-feeding on the alcohol and aldehyde dehydrogenases was examined in the $105,000 \times g$ supernatant fraction of control and aspirin-fed rats to determine if these reactions also were induced by ASA-feeding. As shown in Table 4, the oxidation of $12-\mathrm{OH}-\mathrm{LA}$ was stimulated by only $24 \%$ in the aspirin-fed rats as compared to control rats and strongly suggests the induction of the cytochrome P-450-dependent $\omega$-hydroxylase is responsible for the rapid increase in dicarboxylic acid formation in the aspirin-fed rats.

\section{DISCUSSION}

The fatty acid $\omega$-hydroxylase is catalyzed by a microsomal cytochrome P-450 which was thought to be a noninducible form of this family of hemeprotein monooxygenases (17). However, it was reported that hypolipidemic agents that cause the proliferation of hepatic peroxisomes, such as clofibrate and DEHP, are potent inducers of the fatty acid $\omega$-hydroxylation system (1821 ). Because of the reports that ASA was a peroxisomal proliferator $(22,23)$, this compound was fed to rats at a dose of $1 \%$ (w/w) of their diet. It was observed that ASA-feeding caused a 3fold increase in laurate 12-hydroxylation without stimulating other known cytochrome P-450-mediated reactions. This in- 
crease is less than the induction observed for DEHP which is approximately 6 -fold over control values $(20)$. The increase in the $\omega$-hydroxylase resulted in a rapid utilization of LA and its conversion to the dicarboxylic acid derivative compared to nonASA-fed rats. This increase in the formation of dodecanedioic acid is apparently due to the increase in the $\omega$-hydroxylase as there was only a minor increase in the dehydrogenation reactions that catalyze the oxidation of 12-OH-LA to the DCA derivative in the ASA-fed rats. Previous studies have established the $\omega$ hydroxylation reaction as the rate-limiting step in the $\omega$-oxidation system (14).

This induction of the $\omega$-hydroxylase is of interest because of the recent findings of Tonsgard and Getz (1) and $\mathrm{Ng}$ et al. (2) that unique long-chain length dicarboxylic acids are found in the serum of patients with RS and that they are uncouplers of oxidative phosphorylation (1). Passi et al. (32) also reported that short- and medium-chain length $\left(\mathrm{C}_{8}-\mathrm{C}_{13}\right)$ dicarboxylic acids inhibited mitochondrial function, but there were differences between these two studies as to the type of mitochondrial inhibition that was observed. Further evidence of the toxicity of dicarboxylic acids was presented by Mortensen and Gregersen (33) who observed that the administration of hexadecanedioic acid was toxic to starved rats.

These studies point to the possible role of dicarboxylic acids in mitochondrial dysfunction, the development of RS, and the role of ASA in this disease. It would be of interest to determine if RS patients have an induced fatty acid $\omega$-hydroxylase. Patients that are diagnosed as having RS but who do not have a history of aspirin therapy should be checked for other agents that may cause the induction of the fatty acid $\omega$-hydroxylase, such as DEHP. DEHP is a common plasticizer used in the manufacture of plastics which may migrate from plastic containers and contaminate foods or the water supply $(34,35)$. Another compound that may cause peroxisomal proliferation is valproic acid (36), an antiepileptic agent. Peroxisomal proliferation has been noted in some RS patients $(37,38)$ and it would be interesting to understand the cause of this increase in number of peroxisomes in view of the relationship between compounds that induce the specific activity of the fatty acid $\omega$-hydroxylase and cause peroxisomal proliferation.

Although ASA, DEHP, and clofibrate are potent and rapid inducers of the peroxisomal fatty acid $\beta$-oxidation and microsomal fatty acid $\omega$-hydroxylation systems in rodents, it is not known if they have similar effects in humans. Fatty acid hydroxylation is found in human liver microsomes (27), but the induction of this system in humans has not been studied. The dose of ASA used in this study ( $1 \% \mathrm{w} / \mathrm{w}$ of the total diet) was used because of its success in causing rapid peroxisomal proliferation in rats (22). Further studies are required to establish if pharmacological levels of ASA will induce these enzymic systems in rodent and humans and if arthritic patients who are on ASA therapy have unusual urinary or serum organic acid patterns. The low incidence of individuals who develop RS after a viral infection suggests there may be a genetic predisposition to the effects of ASA or other possible agents that may cause this disease. Further studies are needed to establish the role of the $\omega$-hydroxylation system in Reye's patients and the toxicity of dicarboxylic acids on mitochondrial function.

Acknowledgments. The author thanks Mrs. Carole Panaro and Mrs. Ann Kleist for their excellent assistance and patience in preparing this manuscript and to Mrs. Chris Chance Plumb for her excellent technical help.

\section{REFERENCES}

1. Tonsgard JH. Getz GS 1985 Effect of Reye's Syndrome serum on isolated chinchilla liver mitochondria. $\mathrm{J}$ Clin Invest 76:816-825

2. $\mathrm{Ng} \mathrm{KJ}$, Andresen BD, Hilty MD, Bianchine JR 1983 Identification of long chain dicarboxylic acids in the serum of two patients with Reye's syndrome. J Chromatogr 276:1-10
3. Lawson AM, Chalmers RA, Watts RWE 1976 Urinary organic acid in man. I. Normal patterns. Clin Chem 22:1283-1287

4. Pettersen JE, Jellum E, Eldjarn L 1972 The occurrence of adipic and suberic acid in urine from ketotic patients. Clin Chim Acta 38:17-24

5. Goodman SI, McCabe ERB, Fennessey PV, Mace JW 1980 Multiple acyl-CoA dehydrogenase (glutaric aciduria type II) with transient hypersarcosinemia and sarcosinuria: possible inherited deficiency of an electron transfer flavoprotein. Pediatr Res 14:12-17

6. Sweetman L, Nyhan WL, Trauner DA, Merritt TA, Singh M 1980 Glutaric aciduria type II. J Pediatr 96:1020-1026

7. Mantagos S, Genel M, Tanaka K 1979 Ethylmalonic-adipic aciduria. In vivo and in vitro studies indicating deficiency of activities of multiple acyl-CoA dehydrogenases. J Clin Invest 64:1580-1589

8. Karpati G, Carpenter S, Engel AG, Watters G, Allen J, Rothman S, Klassen G, Mamer OA 1975 The syndrome of systemic carnitine deficiency. Neurology $25: 16-24$

9. Tanaka K 1975 Branched pentanoic acidemia and medium chain dicarboxylic aciduria induced by hypoglycin A: inhibition of several short chain acyl CoA dehydrogenases. In: Kean EA (ed) Hypoglycin. Academic Press, New York, pp 67-92

10. Kolvraa S, Gregersen N, Christensen E, Hobolth N 1982 In vitro fibroblast studies in a patient with $\mathrm{C}_{6}-\mathrm{C}_{10}$-dicarboxylic aciduria: evidence for a defect in general acyl-CoA dehydrogenase. Clin Chim Acta 126:53-67

11. Gregersen N 1985 The acyl-CoA dehydrogenation deficiencies. Scand J Clin Lab Invest 45(suppl 174): 1-60

12. Lu AYH, Junk KW, Coon MJ 1969 Resolution of the cytochrome P-450containing $\omega$-hydroxylation system of liver microsomes into three components. J Biol Chem 244:3714-3721

13. Das ML, Orrenius S, Ernster L 1968 On the fatty acid and hydrocarbon hydroxylation in the rat liver microsomes. Eur J Biochem 4:519-523

14. Gregersen N, Mortensen PB, and Kolvraa S 1983 On the biologic origin of $\mathrm{C}_{6-}$ $\mathrm{C}_{10}$-dicarboxylic and $\mathrm{C}_{6}-\mathrm{C}_{10} \omega$-1-hydroxymonocarboxylic acids in human and rat with acyl-CoA dehydrogenation deficiencies: in vitro studies on the $\omega-$ and $\omega$-1-oxidation of medium-chain $\left(\mathrm{C}_{6}-\mathrm{C}_{10}\right)$ fatty acids in human and rat liver. Pediatr Res 17:828-834

15. Bjorkhem I 1972 On the role of alcohol dehydrogenase in $\omega$-oxidation of fatty acids. Eur J Biochem 30:441-451

16. Bjorkhem I $1973 \omega$-Oxidation of stearic acid in the normal starved and diabetic rat liver. Eur J Biochem 40:415-422

17. Okita RT, Masters BSS 1979 Effect of phenobarbital treatment and cytochrome P-450 inhibitors on the laurate $\omega-$ and $(\omega-1)$-hydroxylase activities of rat liver microsomes. Drug Metab Dispos 8:147-151

18. Orton TC, Parker GL 1982 The effect of hypolipidemic agents on the hepatic microsomal drug-metabolizing enzyme system of the rat. Induction of cytochrome P-450 with specificity toward terminal hydroxylation of lauric acid. Drug Metab Dispos 10:110-115

19. Gibson GG, Orton TC, Tamburini PP 1982 Cytochrome P-450 induction by clofibrate. Biochem J 203:161-168

20. Okita $R$, Chance $C 1984$ Induction of laurate $\omega$-hydroxylase by di-(2-ethylhexyl)phthalate in rat liver microsomes. Biochem Biophys Res Commun 121:304-309

21. Lake BG, Tredger JM, Gray TJB, Stubberfield CR, Hodder KD, Gangolli SD, Williams R 1984 The effect of peroxisome proliferators on the metabolism and spectral interaction of endogenous substrates of cytochrome P-450 in rat hepatic microsomes. Life Sci 35:2621-2626

22. Ishii H, Suga T 1979 Clofibrate-like effects of acetylsalicylic acid on peroxisomes and on hepatic and serum triglyceride levels. Biochem Pharmacol 28:2829-2833

23. Sakurai T, Miyazawa S, Osumi T, Furuta S, Hashimoto $\Upsilon 1981$ Induction of peroxisomal $\beta$-oxidation by the administration of acetylsalicylic acid. Toxicol Appl Pharmacol 59:8-14

24. Halpin TJ, Holtzhauer FJ, Campbell RJ, Hall LJ Correa-Villasenor A, Lanese R, Rice J, Hurwitz ES 1982 Reye's syndrome and medication use. JAMA 248:687-691

25. Waldman RJ, Hall WN, McGee H, Van Amburg G 1982 Aspirin as a risk factor in Reye's syndrome. JAMA 247:3089-3094

26. Hurwitz ES, Barrett MJ, Bregman D Gunn WJ, Schonberger LB, Fairweather WR, Drage JS, LaMontagne JR, Kaslow RA, Burlington B, Quinnan GV, Parker RA, Phillips K, Pinsky P, Dayton D, Dowdle WR 1985 Public Health Service Study on Reye's syndrome and medications. A report of the pilot phase N Engl J Med 313:849-857

27. Okita RT, Jakobsson SW, Prough RA, Masters BSS 1979 Lauric acid hydroxylation in human liver and kidney cortex microsomes. Biochem Pharmacol 28:3385-3390

28. Werringloer J 1978 Assay of formaldehyde generated during microsomal oxidation reactions. Methods Enzymol 52:297-302

29. Prough RA, Burke MD, Mayer RT 1978 Direct fluorometric methods for measuring mixed-function oxidase activity. Methods Enzymol 52:372-377

30. Lazarow PB 1981 Assay of peroxisomal $\beta$-oxidation of fatty acids. Methods Enzymol 72:315-319

31. Kupfer D, Navarro J, Miranda GK, Piccolo DE, Theoharides A 1980 Studies on the oxidation of $\omega$-hydroxyprostaglandins by an NAD-dependent dehydrogenase from mammalian liver cytosol. Arch Biochem Biophys 199:228235

32. Passi S. Picardo M, Nazzaro-Porro M, Breathnach A, Confaloni AM, and Serlupi-Crescenzi G 1984 Antimitochondrial effect of saturated medium chain length $\left(\mathrm{C}_{8}-\mathrm{C}_{13}\right)$ dicarboxylic acids. Biochem Pharmacol 33:103-108 
33. Mortensen PB. Gregersen N 1982 The biological origin of ketotic dicarboxylic aciduria: II. In vivo and in vitro investigations of the $\beta$-oxidation of $\mathrm{C}_{8}-\mathrm{C}_{16}$ dicarboxylic acids. Biochem Biophys Acta 710:477-484

34. Thomas JA. Darby TD. Wallin RF, Garvin PJ. Martis L 1978 A review of the biological effects of di-(2-ethylhexyl)phthalate. Toxicol Appl Pharmacol 45:1-27

35. Turnbull D. Rodricks JV 1985 Assessment of possible carcinogenic risk to humans resulting from exposure to di(2-ethylhexyl)phthalate (DEHP). J Am
Coll Toxicol 4:111-145

36. Horie S. Suga T 1985 Enhancement of peroxisomal $\beta$-oxidation in the liver of rats and mice treated with valproic acid. Biochem Pharmacol 34:1357-1362

37. Partin JC 1974 Liver ultrastructure in Reye's syndrome. In: Pollack JD (ed) Reye's Syndrome I. Grune and Stratton, New York, pp 117-129

38. Partin JC, Bove K, Partin JS, Schubert WK 1978 Liver and muscle ultrastructure in Reye's syndrome. In: Crocker JFS (ed) Reye's Syndrome II. Grune and Stratton, New York, pp 217-236

\title{
Announcements
}

\author{
Annual Meeting
}

The American Pediatric Society and the Society for Pediatric Research announce the abstract deadline for the 1987 Annual Meeting (April 27-May 1, Disneyland Hotel, Anaheim, CA) has been set as December 3, 1986.

For further information contact: SPR - Debbie L. Wogenrich, The Society for Pediatric Research, Department of Pediatrics, UNM School of Medicine, Albuquerque, NM 87131 (505)277-6629 or APS-Dr. Audrey K. Brown, Secretary-Treasurer, Department of Pediatrics, Box 49, SUNY, Health Sciences Center at Brooklyn, 450 Clarkson Avenue, Brooklyn, NY 11203, (718)2701692.

\section{Announcement of Prize Competition for 1987 and 1988}

The Central Association of Swiss Milk Producers, West-strasse 10, CH-3000 Bern 6, announces the competition for the International Award for Modern Nutrition which it is donating for 1987 as well as 1988. The award amounts to SFr. 20'000.--and will be awarded to a scientist from a member state of the International Dairy Federation*.

Topic for 1987: Importance of the Trace-Elements $\mathrm{Zn}, \mathrm{Se}$ and $\mathrm{Cr}$ in Nutrition-Deadline: February 15, 1987.

Topic for 1988: Nutrition and Sport-Deadline: February 15, 1988.

Authors of scientific articles dealing with the topic in question are invited to submit the following documents in triplicate: curriculum vitae; bibliography; reprints of the most relevant articles related to the award-topic, which have been published during the past $5 \mathrm{yr}$.

The documents should be written in English, French or German and sent to the President of the jury, Prof. Dr. med. Ettore Rossi, Medizinische Universitäts-Kinderklinik, Inselspital, CH-3010 Bern/Switzerland.

* Member states are: America, Algeria, Austria, Australia, Belgium, Canada, Chile, Czechoslovakia, Denmark, Finland, France, Greece, W. Germany, Hungary, India, Italy, Iraq. Ireland, Israel, Japan, Kenya, Luxemburg, Malta, Netherlands, Norway, New Zealand, Poland, Spain, Sweden, South Africa, Switzerland, United Kingdom. USSR.

\section{Scientific Meeting}

The Society for Behavioral Pediatrics will hold its 5th Annual Scientific Meeting in Anaheim, CA on April 26-27, 1987 in conjunction with the SPR/APS/APA meetings at the Disneyland Hotel. For further information and registration forms, please contact Ms. Noreen Spota at (215) 248-9168 or Dr. Candace Erickson at (212) 305-9862. 